JOURNAL OF

FUNCTION SPACES AND APPLICATIONS

Volume 6, Number 2 (2008), 187-203 (c) 200*, Scientific Horizon

http://www.jfsa.net

\title{
Convolution on spaces of locally summable functions
}

\author{
Federica Andreano and Renato Grande
}

(Communicated by Jürgen Appell)

2000 Mathematics Subject Classification. 42A75, 42A45.

Keywords and phrases. Marcinkiewicz spaces, convolution, Hilbert transform.

\footnotetext{
Abstract. In this work we prove the existence of convolution on Marcinkiewicz spaces $\mathfrak{M}^{p}(\mathbb{R}), 1 \leq p<\infty$, and, using pointwise approximate identities, we extend the classical definition of Hilbert transform to such spaces.
}

\section{Introduction}

We are interested in extending classical results in the theory of singular integrals to spaces of functions that are only locally summable. The natural environment should be the space $L_{\text {loc }}^{p}(\mathbb{R})$, but the most typical singular integral, the Hilbert transform, does not converge therein. Therefore we need some decay condition. An interesting way to introduce mild decay conditions is based upon boundedness of integral averages over large intervals in $\mathbb{R}$. This leads to Marcinkiewicz spaces $\mathfrak{M}^{p}(\mathbb{R})$ defined in Section 2. These spaces have been studied also in [3] and in [1].

In [1], the author proves that any regular bounded Borel measure gives rise to a bounded convolution operator on $\mathfrak{M}^{p}$, for $p \geq 1$. 
In [3], K.S. Lau studies the convolution operators on $\mathfrak{M}^{p}, p \geq 1$, and on its closed subspace of regular functions, i.e. functions such that

$$
\lim _{T \rightarrow \pm \infty} \int_{T}^{T+a}|f|^{p}=0 \quad \text { for } a>0 .
$$

Lau proves that, if $\mu$ is a bounded regular Borel measure on $\mathbb{R}$, such that

$$
\int_{\mathbb{R}}|x| d|\mu|<+\infty
$$

the convolution operator $\phi_{\mu}$ (defined by $\phi_{\mu}(f)=\mu * f$ ), restricted to the subspace of regular functions of $\mathfrak{M}^{p}$ satisfies

$$
\lim _{T \rightarrow \infty} \frac{1}{2 T} \int_{\mathbb{R}}\left|\left(\chi_{T} \phi_{\mu}-\phi_{\mu} \chi_{T}\right) f\right|^{p}=0,
$$

where $\chi_{T}$ is the characteristic function of $[-T, T]$.

In Section 2, we give a direct proof of the existence of convolution on $\mathfrak{M}^{p}$, for $p \geq 1$ (see Proposition 2.1), even though this also follows from the result in [1].

In Section 3, we provide examples to show that pointwise approximate identities $\varphi_{\alpha}$ do not, in general, converge pointwise: convergence does not even hold at Lebesgue points for $f \in \mathfrak{M}^{p}(\mathbb{R})$ if the least upper monotone bounding function $\psi$ of $\varphi$ is not in $L^{1}(\mathbb{R})$.

In Section 4, we prove the existence of pointwise approximate identities on functions $f \in \mathfrak{M}^{p}(\mathbb{R})$ under the additional hypothesis that the least upper monotone bound of $\varphi$ belongs to $L^{1}(\mathbb{R})$. We devote attention to pointwise convergence only: presumably, approximate identities that converge pointwise converge also in the norm of $\mathfrak{M}^{p}(\mathbb{R})$, but we shall return to this subject in a forthcoming paper.

In Section 5, we make use of the convergence result to show the convergence of the Hilbert transform on $\mathfrak{M}^{p}(\mathbb{R})$.

\section{Convolution on Marcinkiewicz spaces}

Let $\mathfrak{M}^{p}(\mathbb{R})$ be the set of functions $f \in L_{\text {loc }}^{p}(\mathbb{R}), 1 \leq p<\infty$ such that

$$
\limsup _{T \rightarrow+\infty} \frac{1}{2 T} \int_{-T}^{T}|f(s)|^{p} d s<+\infty
$$


$\mathfrak{M}^{p}(\mathbb{R}), 1 \leq p<+\infty$, is a vector space and

$$
\|f\|_{p}^{p}=\limsup _{T \rightarrow+\infty} \frac{1}{2 T} \int_{-T}^{T}|f(s)|^{p} d s
$$

defines a seminorm on $\mathfrak{M}^{p}(\mathbb{R})$.

It is possible to prove that $\mathfrak{M}^{p}(\mathbb{R}), 1 \leq p<+\infty$, is complete with respect to such seminorm (see [6]).

In the following proposition we prove that the convolution of a function in $\mathfrak{M}^{p}(\mathbb{R})$ with a function in $L^{1}(\mathbb{R})$ is finite almost everywhere.

Proposition 2.1. Let $f \in \mathfrak{M}^{p}(\mathbb{R}), 1 \leq p<+\infty$ and $\varphi \in L^{1}(\mathbb{R})$.

Then the integral

$$
\int_{-\infty}^{+\infty} f(x-t) \varphi(t) d t
$$

exists for $x \in \mathbb{R}$ a.e.

Proof. For simplicity, let us take $f, \varphi \geq 0, \varphi \in L^{1}(\mathbb{R}), f \in \mathfrak{M}^{p}(\mathbb{R})$. We prove that the double integral

$$
\frac{1}{2 T} \int_{-T}^{T} \int_{-\infty}^{+\infty} f(x-t) \varphi(t) d t d x
$$

is finite and hence

$$
\int_{-\infty}^{+\infty} f(x-t) \varphi(t) d t<+\infty
$$

for almost all $x \in \mathbb{R}$.

$$
\begin{aligned}
& \frac{1}{2 T} \int_{-T}^{T} \int_{-\infty}^{+\infty} f(x-t) \varphi(t) d t d x \\
& \quad=\frac{1}{2 T} \int_{-\infty}^{+\infty} \varphi(t)\left(\int_{-T}^{T} f(x-t) d x\right) d t \\
& =\lim _{K \rightarrow+\infty} \frac{1}{2 T} \int_{-K}^{K} \varphi(t)\left(\int_{-T}^{T} f(x-t) d x\right) d t \\
& \quad=\lim _{K \rightarrow+\infty} \int_{-K}^{K} \varphi(t)\left(\frac{1}{2 T} \int_{-T-t}^{T-t} f(x) d x\right) d t \\
& \leq \lim _{K \rightarrow+\infty} \int_{-K}^{K} \varphi(t) \frac{1}{2(T+|t|)}\left(\int_{-T-|t|}^{T+|t|} f(x) d x\right) \frac{T+|t|}{T} d t .
\end{aligned}
$$


For sufficiently large $T$, given $\varepsilon>0$, there exists $\widetilde{T}$ such that

$$
\frac{1}{2 \widetilde{T}} \int_{-\widetilde{T}}^{\widetilde{T}} f(x) d x \leq\|f\|_{1}+\varepsilon \quad \text { and } \quad 1+\frac{|t|}{T}<2
$$

(it is sufficient to take $T>\widetilde{T}+K$ ).

Hence

$\lim _{K \rightarrow+\infty} \int_{-K}^{K} \varphi(t) \frac{1}{2(T+|t|)}\left(\int_{-T-|t|}^{T+|t|} f(x) d x\right) \frac{T+|t|}{T} d t \leq 2\|\varphi\|_{L^{1}}\left(\|f\|_{1}+\varepsilon\right)$.

\section{An example where convergence fails}

Let $f$ be a function in the Marcinkiewicz space $\mathfrak{M}^{1}(\mathbb{R})$, or more generally $\mathfrak{M}^{p}(\mathbb{R})$ for $1 \leqslant p<+\infty$. Let $\varphi \in L^{1}(\mathbb{R}), \varphi \geq 0, \int_{-\infty}^{+\infty} \varphi(x) d x=1$, and consider the approximate identity

$$
\left\{\varphi_{\varepsilon}(x)=\frac{1}{\varepsilon} \varphi\left(\frac{x}{\varepsilon}\right): \varepsilon>0\right\} .
$$

Since $f$ is only locally integrable, convergence of the approximate identity is not granted: is it true that, for almost every Lebesgue point $x_{0}$ of $f$ (see Definition 4.6),

$$
\lim _{\varepsilon \rightarrow 0}\left(\varphi_{\varepsilon} * f\right)\left(x_{0}\right)=f\left(x_{0}\right) ?
$$

We show now that this is not always the case by providing a counterexample.

Without loss of generality, we may assume that $x_{0}=0$ is a Lebesgue point: from now on we shall restrict attention to convergence at the point 0 . Let $\chi_{n}$ be the characteristic function of the interval $\left[2^{n}, 2^{n}+1\right]$, and let $\alpha_{n}>0$ be such that

$$
\sum_{n=0}^{+\infty} \alpha_{n}=1
$$

We set

$$
\varphi=\sum_{n=0}^{+\infty} \alpha_{n} \chi_{n} .
$$

Consider now the following function $f \in \mathfrak{M}^{1}(\mathbb{R})$ :

$$
f(x)=\sum_{n=0}^{+\infty} c_{n} \chi_{n}(|x|)
$$


where $c_{n}>0, c_{n}=O\left(2^{n}\right)$. Observe that, for $\mathrm{C}$ constant,

$$
\frac{1}{2^{n+1}} \int_{-2^{n}-1}^{2^{n}+1} f(x) d x<C \frac{1}{2^{n+1}} \sum_{k=0}^{n} 2^{k}<C
$$

hence $f \in \mathfrak{M}^{1}(\mathbb{R})$. Similarly, if we define $f_{p}$ by choosing $c_{n}=O\left(2^{n / p}\right)$, then $f_{p} \in \mathfrak{M}^{p}(\mathbb{R})$.

Proposition 3.1. If $\sum_{m=0}^{+\infty} \alpha_{m} c_{m-n}=+\infty$, then (1) fails. The condition is satisfied, for instance, if $\alpha_{m}=2^{-m}$ and $c_{m}=2^{m}$.

Proof. Let $\varepsilon=2^{-n}$. Then $\chi_{m}\left(\frac{x}{\varepsilon}\right)=\chi_{\left[2^{m-n}, 2^{m-n}+2^{-n}\right]}(x)$. Hence

$$
\varphi_{\varepsilon}(x)=2^{n} \sum_{m=0}^{+\infty} \alpha_{m} \chi_{\left[2^{m-n}, 2^{m-n}+2^{-n}\right]}(x)
$$

Therefore

$$
\begin{aligned}
\left(\varphi_{\varepsilon} * f\right)(0) & =\int_{-\infty}^{+\infty} \varphi_{\varepsilon}(x) f(x) d x \\
& =2^{n} \sum_{m, k=0}^{+\infty} \alpha_{m} c_{k} \chi_{\left[2^{m-n}, 2^{m-n}+2^{-n}\right]}(x) \chi_{\left[2^{k}, 2^{k}+1\right]}(x) \\
& =2^{n} \sum_{m=n}^{+\infty} \alpha_{m} c_{m-n} \chi_{\left[2^{m-n}, 2^{m-n}+2^{-n}\right]}(x)
\end{aligned}
$$

Then

$$
\int_{-\infty}^{+\infty} \varphi_{\varepsilon}(x) f(x) d x=\sum_{m=n}^{+\infty} \alpha_{m} c_{m-n}=+\infty
$$

Remark 3.2. In what follows, it will be of interest to relate the function $\varphi$ of this counterexample to its least upper bound

$$
\psi(x)=\operatorname{supess}_{|t| \geqslant|x|}|\varphi(t)| .
$$

In particular, we observe that, with $\varphi$ chosen as in Proposition 3.1, if we assume $\left\{\alpha_{n}\right\}$ non-increasing, the function $\psi$ does not satisfy the following properties:

(1) $\psi \in L^{1}(\mathbb{R})$

(2) $\lim _{x \rightarrow \pm \infty} x \psi(x)=0$.

Indeed, $\psi(x)=\alpha_{n}$ if $2^{n-1}+1<x \leqslant 2^{n}+1$. As a consequence, condition (1) is satisfied if and only if the series $\sum_{n=0}^{+\infty} 2^{n} \alpha_{n}$ is convergent, and condition (2) if and only if its general term $2^{n} \alpha_{n}$ vanishes at infinity. 
More generally, notice that, by Proposition 3.1, if condition (1) is not satisfied, convergence fails in (1). Observe that neither condition is satisfied if, for instance, we choose $\alpha_{n}=2^{-n}, n=1,2, \ldots$, both are satisfied if $\alpha_{n}=C 2^{-n} / n^{2}$ (here the normalization constant $C$ is determined by the condition $\sum_{0}^{\infty} \alpha_{n}=1$ ), and only the second one is satisfied if $\alpha_{n}=C^{\prime} 2^{-n} / n$. Therefore the second condition cannot imply convergence in (1), and we are led to investigate whether the first condition implies convergence.

\section{A sufficient condition for pointwise convergence}

Proposition 4.1. Let $\varphi, \psi \in L^{1}(\mathbb{R})$ (where $\psi$ is defined as in Remark 3.2). Then the family $\left\{\varphi_{\varepsilon}\right\}_{\varepsilon>0}$, where

$$
\varphi_{\varepsilon}(t)=\frac{1}{\varepsilon} \varphi\left(\frac{t}{\varepsilon}\right)
$$

is an approximate identity on $\mathfrak{M}^{p}(\mathbb{R})$, i.e. for $\varepsilon \rightarrow 0^{+}$

$$
\left(f * \varphi_{\varepsilon}\right)\left(x_{o}\right) \rightarrow f\left(x_{o}\right) \int_{-\infty}^{+\infty} \varphi(t) d t \quad \text { for a.a. } x_{o} \in \mathbb{R} .
$$

(See [8] for an analogous theorem in the case $f \in L^{p}(\mathbb{R})$ ).

To prove Proposition 4.1 we need several lemmas.

Lemma 4.2. For $1<p<+\infty, \mathfrak{M}^{p}(\mathbb{R}) \subseteq \mathfrak{M}^{1}(\mathbb{R})$ and $\|f\|_{1} \leq\|f\|_{p}$.

Proof. Let $f \in \mathfrak{M}^{p}(\mathbb{R})$. By Jensen's inequality we have that

$$
\left(\frac{1}{2 T} \int_{-T}^{T}|f(t)| d t\right)^{p} \leq \frac{1}{2 T} \int_{-T}^{T}|f(t)|^{p} d t .
$$

Since $f \in \mathfrak{M}^{p}(\mathbb{R})$,

$$
\limsup _{T \rightarrow+\infty} \frac{1}{2 T} \int_{-T}^{T}|f(t)|^{p} d t=\|f\|_{p}^{p}
$$

Hence

$$
\begin{aligned}
\|f\|_{1}^{p} & =\left(\limsup _{T \rightarrow+\infty} \frac{1}{2 T} \int_{-T}^{T}|f(t)| d t\right)^{p} \\
& =\limsup _{T \rightarrow+\infty}\left(\frac{1}{2 T} \int_{-T}^{T}|f(t)| d t\right)^{p}
\end{aligned}
$$




$$
\leq \limsup _{T \rightarrow+\infty} \frac{1}{2 T} \int_{-T}^{T}|f(t)|^{p} d t=\|f\|_{p}^{p} .
$$

We shall need the following easy facts:

Lemma 4.3. Translation is continuous on $\mathfrak{M}^{p}(\mathbb{R})$.

Lemma 4.4. Let $\varphi, \psi \in L^{1}(\mathbb{R})$ (where $\psi$ is defined as in Remark 3.2). Then

$$
\lim _{|x| \rightarrow+\infty} \varphi(x)=0 .
$$

Proof. If $0 \leq x_{1} \leq x_{2}$, by the definition of $\psi(x), \psi\left(x_{1}\right) \geq \psi\left(x_{2}\right)$.

Hence $\psi$ is monotone not-increasing for $0 \leq x$. Since $\psi \in L^{1}(\mathbb{R})$, it follows that

We obviously have that

$$
\lim _{x \rightarrow+\infty} \psi(x)=0
$$

$$
|\varphi| \leq \psi
$$

and therefore the statement for $x \rightarrow+\infty$.

The same holds for $x \rightarrow-\infty$, since $\psi$ is even.

The next statement requires more work

Lemma 4.5. Under the same hypotheses of Lemma 4.4, the function $r \psi(r)$ is uniformly bounded on $\mathbb{R}_{+}$and

$$
\lim _{r \rightarrow 0^{+}} r \psi(r)=0, \quad \lim _{r \rightarrow+\infty} r \psi(r)=0 .
$$

Proof. $\psi \in L^{1}(\mathbb{R})$ and therefore

$$
\int_{r<x<2 r} \psi(x) d x \rightarrow 0
$$

as $r \rightarrow 0^{+}$and $r \rightarrow+\infty$.

By the integral mean theorem there exists $\xi_{r}$ such that $r<\xi_{r}<2 r$ and

$$
J_{r}=\int_{r<x<2 r} \psi(x) d x=r \psi\left(\xi_{r}\right) .
$$

Hence

$$
r \psi(2 r) \leq J_{r} \leq r \psi(r)
$$

and since $J_{r} \rightarrow 0$ as $r \rightarrow 0^{+}$and $r \rightarrow+\infty$, it follows that the same holds for $r \psi(r)$.

Furthermore

$$
r \psi(2 r)<\int_{r<x<2 r} \psi(x) d x \leq \int_{\mathbb{R}} \psi(x) d x
$$


and so $r \psi(r)$ is bounded in $\mathbb{R}_{+}$(and hence in $\mathbb{R}$ ).

At this point it is useful to remind the following

Definition 4.6. Let $g \in L^{1}(a, b)$. A point $t_{o} \in[a, b]$ such that the limit

$$
\lim _{h \rightarrow 0} \frac{1}{h} \int_{t_{o}}^{t_{o}+h}\left|g(s)-g\left(t_{o}\right)\right| d s, \quad t_{o}+h \in[a, b]
$$

exists and is equal to zero is called a Lebesgue point for $g$.

Remark 4.7. If $g \in L^{1}(a, b)$, a.a. $t_{o} \in[a, b]$ satisfy the condition of being Lebesgue points of $g$.

Lemma 4.8. Let $f \in \mathfrak{M}^{p}(\mathbb{R})$ and $x_{o}$ a Lebesgue point for $f$. Let $\varphi \in L^{1}(\mathbb{R})$ and let $\psi_{\varepsilon} \in L^{1}(\mathbb{R})$, where $\psi_{\varepsilon}$ is defined as in Proposition 4.1. Then for all $\tilde{\varepsilon}>0 \exists \delta=\delta(\tilde{\varepsilon})$ such that

$$
K_{\delta}=\int_{-\delta}^{\delta} \int_{0}^{t}\left|f\left(x_{o}-s\right)-f\left(x_{o}\right)\right| d s d \psi_{\varepsilon}(t)<c \tilde{\varepsilon},
$$

for $c>0$ constant.

Proof. Since $x_{o}$ is a Lebesgue point, the function

$$
t \rightarrow \int_{0}^{t}\left|f\left(x_{o}-s\right)-f\left(x_{o}\right)\right| d s
$$

is $o(t)$ : hence for all $\tilde{\varepsilon}$ there exists $\delta=\delta\left(\tilde{\varepsilon}, x_{o}\right)$ such that for $|t|<\delta$

$$
\int_{0}^{t}\left|f\left(x_{o}-s\right)-f\left(x_{o}\right)\right| d s<\tilde{\varepsilon} t
$$

and hence

$$
K_{\delta} \leq \int_{-\delta}^{\delta} \tilde{\varepsilon} t d \psi_{\varepsilon}(t)
$$

Integrating by parts, the right-handside becomes

$$
\begin{aligned}
\int_{-\delta}^{\delta} \tilde{\varepsilon} t d \psi_{\varepsilon}(t) & =\left.\tilde{\varepsilon} t \psi_{\varepsilon}(t)\right|_{-\delta} ^{\delta}-\tilde{\varepsilon} \int_{-\delta}^{\delta} \psi_{\varepsilon}(t) d t \\
& =\tilde{\varepsilon} \delta \psi_{\varepsilon}(\delta)+\tilde{\varepsilon} \delta \psi_{\varepsilon}(-\delta)-\tilde{\varepsilon} \int_{-\delta}^{\delta} \psi_{\varepsilon}(t) d t .
\end{aligned}
$$

Since $\psi_{\varepsilon}$ is even and non-negative, we get

$$
K_{\delta} \leq 2 \tilde{\varepsilon} \delta \psi_{\varepsilon}(\delta)+2 \tilde{\varepsilon} \int_{0}^{+\infty} \psi_{\varepsilon}(t) d t .
$$


By Lemma 4.5 we have that

$$
\delta \psi_{\varepsilon}(\delta)=\frac{\delta}{\varepsilon} \psi\left(\frac{\delta}{\varepsilon}\right)
$$

is uniformly bounded and hence

$$
K_{\delta} \leq 2\left(c^{\prime}+\|\psi\|_{1}\right) \tilde{\varepsilon}=c \tilde{\varepsilon} .
$$

Lemma 4.9. Let $f \in \mathfrak{M}^{p}(\mathbb{R})$. If $x_{o}$ is a Lebesgue point of $f$,

$$
\int_{0}^{t}\left|f\left(x_{o}-s\right)-f\left(x_{o}\right)\right| d s \leq c|t|
$$

where $c$ is a constant depending on $\delta$ (same $\delta$ as in Lemma 4.8).

Proof. It is sufficient to take $|t|>\delta$, since we need the inequality only for such $t$ in order to prove Proposition 4.1. Nevertheless the statement of the Lemma holds also for $|t| \leq \delta$, by Lebesgue property.

Let $|t|>\delta$.

$$
\int_{0}^{t}\left|f\left(x_{o}-s\right)-f\left(x_{o}\right)\right| d s \leq \int_{0}^{t}\left|f\left(x_{o}-s\right)\right| d s+|t|\left|f\left(x_{o}\right)\right| .
$$

By Lemma 4.2, $f \in \mathfrak{M}^{1}(\mathbb{R})$ and by Lemma 4.3 translation is continuous with respect to the $\mathfrak{M}^{1}-$ norm. Hence from the definition of $\mathfrak{M}^{1}-$ norm, it follows that there exists a constant $c(\delta)$ depending on $\delta$ such that

$$
\int_{0}^{t}\left|f\left(x_{o}-s\right)\right| d s \leq c(\delta)|t|
$$

and therefore the statement holds.

Proof of Proposition 4.1. We must show that

$$
\left|\int_{-\infty}^{+\infty} f\left(x_{o}-t\right) \varphi_{\varepsilon}(t) d t-f\left(x_{o}\right)\right| \rightarrow 0
$$

as $\varepsilon \rightarrow 0^{+}$.

We may take $x_{o}$ a Lebesgue point for $f$, i.e.

$$
\int_{0}^{t}\left|f\left(x_{o}+s\right)-f\left(x_{o}\right)\right| d s \leq \tilde{\varepsilon}|s|, \quad \text { for all } t:|t|<\delta=\delta(\tilde{\varepsilon}) .
$$

Let us suppose that

$$
\int_{-\infty}^{+\infty} \varphi(t) d t \neq 0
$$


The reader may easily verify that the same arguments hold in the case

$$
\int_{-\infty}^{+\infty} \varphi(t) d t=0
$$

For simplicity we set

$$
\int_{-\infty}^{+\infty} \varphi(t) d t=1
$$

If $\delta=\delta(\tilde{\varepsilon})$, we have

$$
\begin{aligned}
\left|\int_{-\infty}^{+\infty} f\left(x_{o}-t\right) \varphi_{\varepsilon}(t) d t-f\left(x_{o}\right)\right| \leq & \int_{-\infty}^{+\infty}\left|f\left(x_{o}-t\right)-f\left(x_{o}\right)\right|\left|\varphi_{\varepsilon}(t)\right| d t \\
\leq & \int_{-\infty}^{-\delta}\left|f\left(x_{o}-t\right)-f\left(x_{o}\right)\right| \psi_{\varepsilon}(t) d t \\
& +\int_{\delta}^{+\infty}\left|f\left(x_{o}-t\right)-f\left(x_{o}\right)\right| \psi_{\varepsilon}(t) d t \\
& +\int_{-\delta}^{\delta}\left|f\left(x_{o}-t\right)-f\left(x_{o}\right)\right| \psi_{\varepsilon}(t) d t \\
= & I_{1}+I_{2}+I_{3} .
\end{aligned}
$$

Let us estimate the third integral first.

$$
I_{3}=\int_{-\delta}^{\delta}\left(\frac{d}{d t} \int_{0}^{t}\left|f\left(x_{o}-s\right)-f\left(x_{o}\right)\right| d s\right) \psi_{\varepsilon}(t) d t .
$$

Integrating by parts and reminding that

$$
\int_{0}^{t}\left|f\left(x_{o}-s\right)-f\left(x_{o}\right)\right| d s<\tilde{\varepsilon}|t| \quad \text { if }|t|<\delta,
$$

we get that

$$
I_{3} \leq 2 \tilde{\varepsilon} \delta \psi_{\varepsilon}(\delta)-\int_{|t|<\delta} \int_{0}^{t}\left|f\left(x_{o}-s\right)-f\left(x_{o}\right)\right| d s d \psi_{\varepsilon}(t),
$$

since $\psi_{\varepsilon}$ is even.

By Lemma $4.5, \delta \psi_{\varepsilon}(\delta)$ is uniformly bounded and it tends to zero as $\varepsilon \rightarrow 0^{+}$. By Lemma 4.8

$$
\int_{|t|<\delta} \int_{0}^{t}\left|f\left(x_{o}-s\right)-f\left(x_{o}\right)\right| d s d \psi_{\varepsilon}(t)<\tilde{C} \tilde{\varepsilon} .
$$


Hence

$$
I_{3} \leq C \tilde{\varepsilon}+\tilde{C} \tilde{\varepsilon}=C^{\prime} \tilde{\varepsilon}
$$

Let us estimate $I_{2}$ now.

We need to show that for any $\tilde{\varepsilon}$

$$
\int_{\delta}^{+\infty}\left|f\left(x_{o}-t\right)-f\left(x_{o}\right)\right| \psi_{\varepsilon}(t) d t \leq C^{\prime \prime} \tilde{\varepsilon}
$$

if $\varepsilon$ is sufficiently small.

We write, as before

$$
I_{2}=\int_{\delta}^{+\infty}\left(\frac{d}{d t} \int_{0}^{t}\left|f\left(x_{o}-s\right)-f\left(x_{o}\right)\right| d s\right) \psi_{\varepsilon}(t) d t .
$$

Integrating by parts we get that

$$
\begin{aligned}
I_{2}= & \left.\int_{0}^{t}\left|f\left(x_{o}-s\right)-f\left(x_{o}\right)\right| d s \psi_{\varepsilon}(t)\right|_{t=\delta} ^{t=+\infty} \\
& -\int_{\delta}^{+\infty} \int_{0}^{t}\left|f\left(x_{o}-s\right)-f\left(x_{o}\right)\right| d s d \psi_{\varepsilon}(t) .
\end{aligned}
$$

In the first term the limit as $t \rightarrow+\infty$ is equal to zero by Lemma 4.9 and Lemma 4.5.

Hence

$$
\begin{aligned}
\left.\int_{0}^{t}\left|f\left(x_{o}-s\right)-f\left(x_{o}\right)\right| d s \psi_{\varepsilon}(t)\right|_{t=\delta} ^{t=+\infty} & =-\int_{0}^{\delta}\left|f\left(x_{o}-s\right)-f\left(x_{o}\right)\right| d s \psi_{\varepsilon}(\delta) \\
& \leq \tilde{\varepsilon} \delta \psi_{\varepsilon}(\delta),
\end{aligned}
$$

since $x_{o}$ is a Lebesgue point for $f$.

Therefore

$$
\left.\int_{0}^{t}\left|f\left(x_{o}-s\right)-f\left(x_{o}\right)\right| d s \psi_{\varepsilon}(t)\right|_{t=\delta} ^{t=+\infty} \leq C \tilde{\varepsilon},
$$

for some $C>0$, by Lemma 4.5. Let us consider now the second term in (3), i.e.

$$
\int_{\delta}^{+\infty} \int_{0}^{t}\left|f\left(x_{o}-s\right)-f\left(x_{o}\right)\right| d s d \psi_{\varepsilon}(t)
$$

By Lemma 4.9,

$$
\int_{\delta}^{+\infty} \int_{0}^{t}\left|f\left(x_{o}-s\right)-f\left(x_{o}\right)\right| d s d \psi_{\varepsilon}(t) \leq c_{\delta} \int_{\delta}^{+\infty}|t| d \psi_{\varepsilon}(t)
$$




$$
\leq C \tilde{\varepsilon}+c_{\delta} \int_{\frac{\delta}{\varepsilon}}^{+\infty} \psi(t) d t .
$$

Since $\varepsilon \rightarrow 0^{+}$, we may choose $\varepsilon$ small enough to get, for fixed $\tilde{\varepsilon}$ (and hence fixed $\delta$ ),

$$
c_{\delta} \int_{\frac{\delta}{\varepsilon}}^{+\infty} \psi(t) d t<\tilde{C} \tilde{\varepsilon},
$$

remembering that $\psi \in L^{1}(\mathbb{R})$.

We get hence that

$$
I_{2}<2 C^{\prime} \tilde{\varepsilon}
$$

for sufficiently small $\varepsilon$.

The estimate of $I_{1}$ is analogous to that of $I_{2}$. Hence for all $\tilde{\varepsilon}>0$ there exists $M$ such that if $\varepsilon$ is sufficiently small

$$
\left|\int_{-\infty}^{+\infty} f\left(x_{o}-t\right) \psi_{\varepsilon}(t) d t-f\left(x_{o}\right)\right|<M \tilde{\varepsilon}
$$

where $M$ depends on $\delta$ (and hence on $\tilde{\varepsilon}$ ). $M$ and $\delta$ depend on $x_{o}$, but they can be chosen bounded if $x_{o}$ lies in a set whose complement has arbitrary small measure.

Therefore the left-handside in (4) tends to zero as $\varepsilon \rightarrow 0^{+}$:

for all $\varepsilon>0$ there exists $\tilde{\varepsilon}>0$ such that

$$
\left|\int_{-\infty}^{+\infty} f\left(x_{o}-t\right) \psi_{\varepsilon}(t) d t-f\left(x_{o}\right)\right|<M \tilde{\varepsilon}
$$

The case

$$
\int_{-\infty}^{+\infty} \varphi(t) d t=0
$$

can be handled analogously.

\section{The Hilbert Transform}

Our aim is to introduce a Hilbert transform operator on Marcinkiewicz spaces. Such an operator should be defined, as in [7], by

$$
H f(x)=\lim _{y \rightarrow 0^{+}} \frac{1}{\pi} \int_{|t| \geq y} \frac{f(x+t)}{t} d t .
$$

If $f \in L^{1}(\mathbb{R})$ the convergence of the integral and the norm boundedness of $H$ are related to the theory of principal value distribution and singular integrals. Here, however, there is a further problem, because, in general, $f$ 
is only locally $L^{1}$ (or $L^{p}$ ), and so the integral might diverge as $t$ becomes large.

In this section we prove that $H$ is well defined on a subset of $\mathfrak{M}^{p}(\mathbb{R})$. For this goal, we must approximate the integral in (5) by finite truncations

$$
H_{N, y} f(x)=\frac{1}{\pi} \int_{N \geq|t| \geq y} \frac{f(x+t)}{t} d t .
$$

Proposition 5.1 (cf. [4]). Let $f \in \mathfrak{M}^{p}(\mathbb{R}), 1 \leq p<+\infty$ be such that for all $x \in \mathbb{R}$

$$
\left|\int_{0}^{t} f(x+\widetilde{t}) d \widetilde{t}\right| \leq C|t|^{\alpha}
$$

for a fixed $\alpha \in(0,1)$. Then the limit

$$
\lim _{N \rightarrow+\infty} \frac{1}{\pi i} \int_{-N}^{N} \frac{f(t)}{t-z} d t, z \in \mathbb{C}_{+}=\{z=x+i y \in \mathbb{C}: y>0\},
$$

exists uniformly on compact subsets of $\mathbb{C}_{+}$.

Proof. Let us set

$$
F(z, N)=\frac{1}{\pi i} \int_{-N}^{N} \frac{f(t)}{t-z} d t
$$

For $N^{\prime \prime}>N^{\prime}$

$$
\begin{aligned}
F\left(z, N^{\prime \prime}\right)-F\left(z, N^{\prime}\right) & =\frac{1}{\pi i} \int_{-N^{\prime \prime}}^{N^{\prime \prime}} \frac{f(t)}{t-z} d t-\frac{1}{\pi i} \int_{-N^{\prime}}^{N^{\prime}} \frac{f(t)}{t-z} d t \\
& =\frac{1}{\pi i} \int_{-N^{\prime \prime}}^{-N^{\prime}} \frac{f(t)}{t-z} d t+\frac{1}{\pi i} \int_{N^{\prime}}^{N^{\prime \prime}} \frac{f(t)}{t-z} d t \\
& =I_{1}+I_{2} .
\end{aligned}
$$

Let us give an estimate of the integral

$$
I_{2}=\frac{1}{\pi i} \int_{N^{\prime}}^{N^{\prime \prime}} \frac{f(t)}{t-z} d t
$$

We have that

$$
\begin{aligned}
\int_{N^{\prime}}^{N^{\prime \prime}} \frac{f(t)}{t-z} d t & =\int_{N^{\prime}}^{N^{\prime \prime}} \frac{f(t)}{(t-x)-i y} d t \\
& =\int_{N^{\prime}-x}^{N^{\prime \prime}-x} \frac{f(x+s)}{s-i y} d s \\
& =\int_{N^{\prime}-x}^{N^{\prime \prime}-x} \frac{\frac{d}{d t} \int_{0}^{t} f(x+s) d s}{t-i y} d t
\end{aligned}
$$




$$
=\left.\frac{\int_{0}^{t} f(x+s) d s}{t-i y}\right|_{N^{\prime}-x} ^{N^{\prime \prime}-x}+\int_{N^{\prime}-x}^{N^{\prime \prime}-x} \frac{\int_{0}^{t} f(x+s) d s}{(t-i y)^{2}} d t .
$$

Hence

$$
\begin{aligned}
& \left|\frac{1}{\pi i} \int_{N^{\prime}}^{N^{\prime \prime}} \frac{f(t)}{t-z} d t\right| \\
& \leq \frac{1}{\pi}\left(\frac{\left|\int_{0}^{N^{\prime \prime}-x} f(x+s) d s\right|}{\left|\left(N^{\prime \prime}-x\right)-i y\right|}+\frac{\left|\int_{0}^{N^{\prime}-x} f(x+s) d s\right|}{\left|\left(N^{\prime}-x\right)-i y\right|}\right. \\
& \left.\quad+\int_{N^{\prime}-x}^{N^{\prime \prime}-x} \frac{\left|\int_{0}^{t} f(x+s) d s\right|}{|t-i y|^{2}} d t\right) \\
& \leq \frac{1}{\pi}\left(\frac{C\left|N^{\prime \prime}-x\right|^{\alpha}}{\sqrt{\left(N^{\prime \prime}-x\right)^{2}+y^{2}}}+\frac{C\left|N^{\prime}-x\right|^{\alpha}}{\sqrt{\left(N^{\prime}-x\right)^{2}+y^{2}}}+\int_{N^{\prime}-x}^{N^{\prime \prime}-x} \frac{C|t|^{\alpha}}{t^{2}+y^{2}} d t\right) .
\end{aligned}
$$

Since $|x| \leq T, \quad y \geq y_{0}>0$ one may choose $N^{\prime}, N^{\prime \prime}$ such that the last quantity becomes less than $\frac{\varepsilon}{2}$. The integral $I_{2}$ may be estimated in an analogous way.

Remark 5.2. By Proposition 5.1 the function

$$
F(z)=\frac{1}{\pi i} \int_{-\infty}^{+\infty} \frac{f(t)}{t-z} d t=\lim _{N \rightarrow+\infty} \frac{1}{\pi i} \int_{-N}^{N} \frac{f(t)}{t-z} d t, \quad z \in \mathbb{C}_{+}
$$

is holomorphic in $\mathbb{C}_{+}$.

Proposition 5.3. In the same hypotheses of Proposition 5.1 the limit

$$
\lim _{N \rightarrow+\infty} \frac{1}{\pi} \int_{N \geq|t| \geq y} \frac{f(x+t)}{t} d t=\lim _{N \rightarrow+\infty} H_{N, y} f(x)=H_{y} f(x)
$$

exists for all $x \in \mathbb{R}$ and for all $y>0$.

Proof.

$$
\int_{N \geq|t| \geq y} \frac{f(x+t)}{t} d t=\int_{y}^{N} \frac{f(x+t)}{t} d t+\int_{-N}^{-y} \frac{f(x+t)}{t} d t .
$$

Let us prove that

$$
\lim _{N \rightarrow+\infty} \int_{y}^{N} \frac{f(x+t)}{t} d t .
$$


exists.

We have that

$$
\begin{aligned}
\int_{y}^{N^{\prime \prime}} & \frac{f(x+s)}{s} d s-\int_{y}^{N^{\prime}} \frac{f(x+s)}{s} d s \\
& =\int_{N^{\prime}}^{N^{\prime \prime}} \frac{f(x+s)}{s} d s \\
& =\int_{N^{\prime}}^{N^{\prime \prime}} \frac{\frac{d}{d t} \int_{0}^{t} f(x+s) d s}{t} d t \\
& =\left.\frac{\int_{0}^{t} f(x+s) d s}{t}\right|_{N^{\prime}} ^{N^{\prime \prime}}+\int_{N^{\prime}}^{N^{\prime \prime}} \frac{\int_{0}^{t} f(x+s) d s}{t^{2}} d t .
\end{aligned}
$$

It follows that

$$
\begin{aligned}
& \left|\int_{y}^{N^{\prime \prime}} \frac{f(x+s)}{s} d s-\int_{y}^{N^{\prime}} \frac{f(x+s)}{s} d s\right| \\
& \quad \leq \quad\left(\frac{C\left|N^{\prime \prime}\right|^{\alpha}}{\left|N^{\prime \prime}\right|}+\frac{C\left|N^{\prime}\right|^{\alpha}}{\left|N^{\prime}\right|}+\int_{N^{\prime}}^{N^{\prime \prime}} \frac{C|t|^{\alpha}}{t^{2}} d t\right)
\end{aligned}
$$

and we may conclude as usual.

Hence, by Proposition 5.3, we get the existence of the limit

$$
\begin{aligned}
H_{y} f(x) & =\lim _{N \rightarrow+\infty} \frac{1}{\pi} \int_{N \geq|t| \geq y} \frac{f(x+t)}{t} d t \\
& =\frac{1}{\pi} \int_{|t| \geq y} \frac{f(x+t)}{t} d t .
\end{aligned}
$$

On the other hand, by the Fatou-Privalov theorem (cfr. [2] and [5]) we have that the limit

$$
\lim _{y \rightarrow 0^{+}} \frac{1}{\pi} \int_{-\infty}^{+\infty} \frac{t f(x+t)}{t^{2}+y^{2}} d t
$$

exists for $x \in \mathbb{R}$ a.e..

Set

$$
v(x, y)=\frac{1}{\pi} \int_{-\infty}^{+\infty} \frac{t f(x+t)}{t^{2}+y^{2}} d t .
$$

Then we know that

$$
\lim _{y \rightarrow 0^{+}} v(x, y)
$$

exists for $x \in \mathbb{R}$ a.e.. 
Proposition 5.4. In the same hypotheses of Proposition 5.1 we get that

$$
\lim _{y \rightarrow 0^{+}}\left|v(x, y)-H_{y} f(x)\right|=0
$$

$x \in \mathbb{R}$ a.e.

Proof. Let us define the function

$$
\Phi(t)= \begin{cases}\frac{t}{t^{2}+1}-\frac{1}{t}, & |t| \geq 1 \\ \frac{t}{t^{2}+1}, & |t|<1 .\end{cases}
$$

Let us observe that $\Phi \in L^{1}(\mathbb{R})$, and that

$$
\Psi(x)=\operatorname{supess}_{|\mathrm{t}| \geq|\mathrm{x}|}|\Phi(t)| \in L^{1}(\mathbb{R})
$$

since

$$
\Psi(x)=\operatorname{supess}_{|\mathrm{t}| \geq|\mathrm{x}|}|\Phi(t)|= \begin{cases}\frac{1}{|x|\left(x^{2}+1\right)}, & |x| \geq 1 \\ \frac{1}{2}, & |x|<1\end{cases}
$$

We have then

$$
\begin{aligned}
v(x, y)-H_{y} f(x) & =\frac{1}{\pi} \int_{-\infty}^{+\infty} \frac{t f(x+t)}{t^{2}+y^{2}} d t-\frac{1}{\pi} \int_{|t| \geq y} \frac{f(x+t)}{t} d t \\
& =\frac{1}{\pi} \int_{-\infty}^{+\infty} f(x+t) \Phi_{y}(t) d t
\end{aligned}
$$

Then, by Proposition 4.1 we get that

$$
\lim _{y \rightarrow 0^{+}} \int_{-\infty}^{+\infty} f(x+t) \Phi_{y}(t) d t=0
$$

and hence the assertion.

Corollary 5.5. In the same hypotheses of Proposition 5.1 the Hilbert transform, defined as

$$
\lim _{y \rightarrow 0^{+}} H_{y} f(x)=H f(x)
$$

exists for $x \in \mathbb{R}$ a.e..

Acknowledgements. The authors would like to thank Massimo Picardello for precious comments and suggestions. 


\section{References}

[1] J. Bertrandias, Espaces de fonctions bornées et continues en moyenne asymptotique d'ordre p, Bull. Soc. Math. France, 5 (1966).

[2] J. B. Garnett, Bounded Analytic Functions, Academic Press, 1981

[3] K. Lau, The class of convolution operators on the Marcinkiewicz spaces, Ann. Inst. Fourier (Grenoble), 31 (1981), 225-243.

[4] B. M. Levitan, Almost Periodic Functions, Gostekhizdat, Moscow, 1953 (Russian).

[5] V. P. Khavin and N. K. Nikol'skij (eds.), Commutative Harmonic Analysis, Encyclopaedia of Mathematical Sciences, Vol. 15, Springer Verlag, 1991.

[6] J. Marcinkiewicz, Un remarque sur les éspaces de A.S. Besicovitch, C.R. Acad. Sc., Paris, t. 208 (1939), 157-159.

[7] E. M. Stein, Singular Integrals and Differentiability Properties of Functions, Princeton University Press, Princeton, 1970

[8] E. M. Stein and G. Weiss, Introduction to Fourier Analysis on Euclidean Spaces, Princeton University Press, Princeton, 1971

Dipartimento di Informatica

Università dell' Insubria

via Mazzini 5

21100 Varese

(E-mail : federica.andreano@uninsubria.it)

Dipartimento di Metodi e Modelli Matematici per le Scienze Applicate Università di Roma "La Sapienza"

via A. Scarpa 16

00161 Rome

(E-mail : grande@dmmm.uniroma1.it)

(Received : July 2006) 


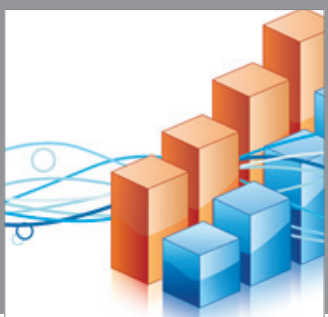

Advances in

Operations Research

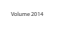

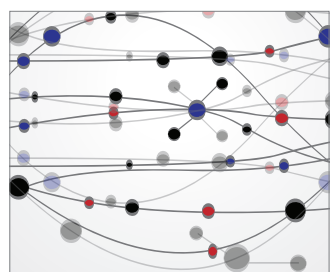

\section{The Scientific} World Journal
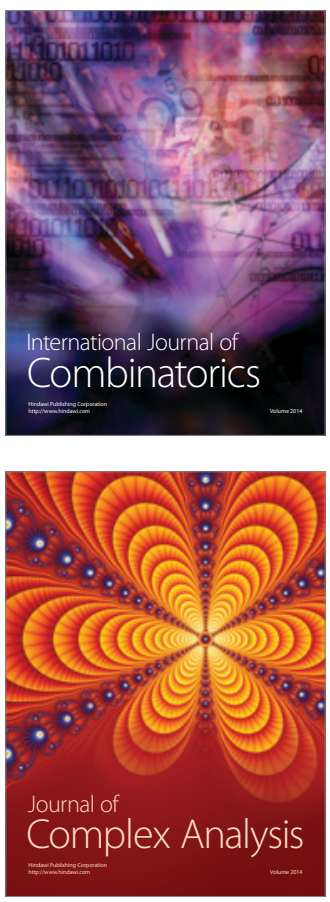

International Journal of

Mathematics and

Mathematical

Sciences
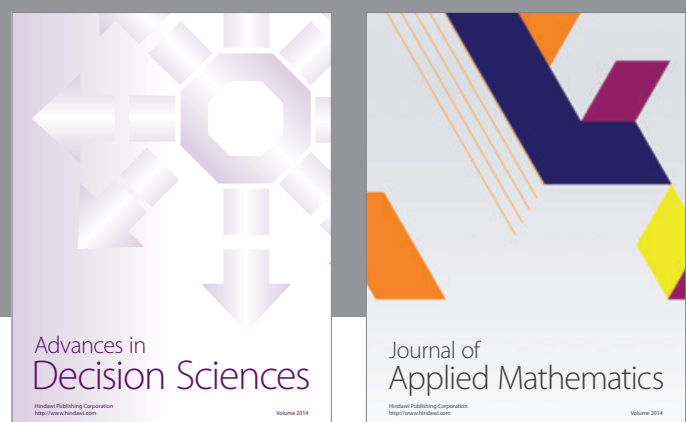

Journal of

Applied Mathematics
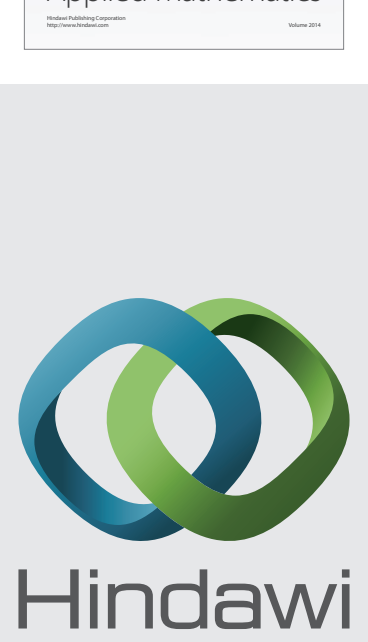

Submit your manuscripts at http://www.hindawi.com
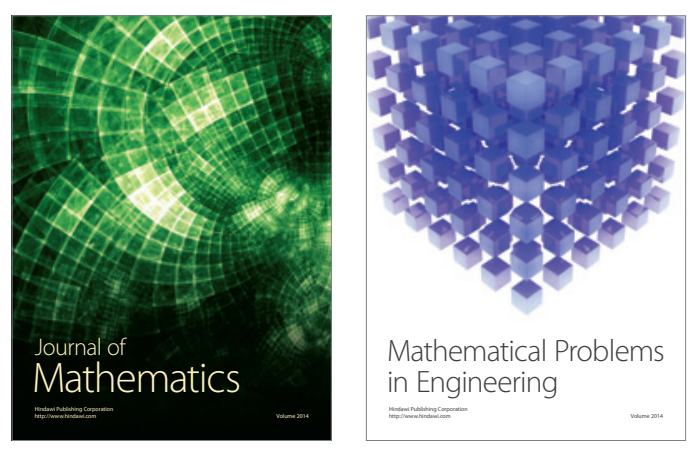

Mathematical Problems in Engineering
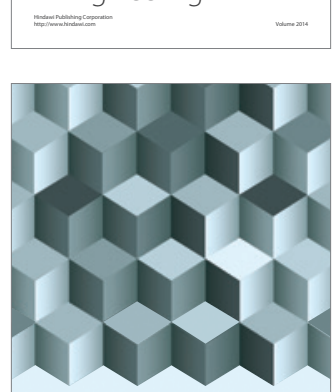

Journal of

Function Spaces
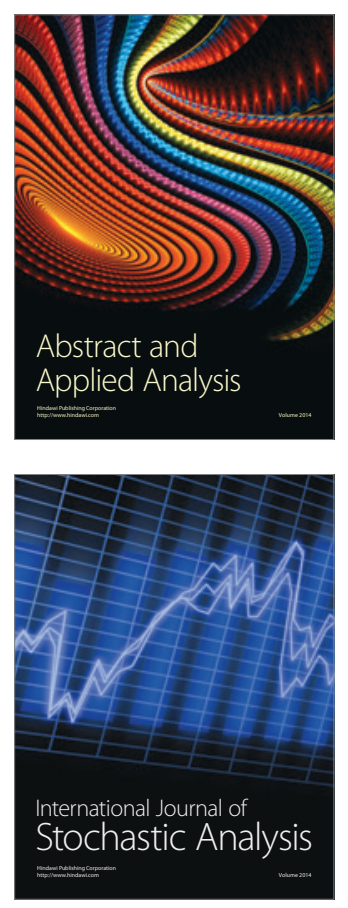

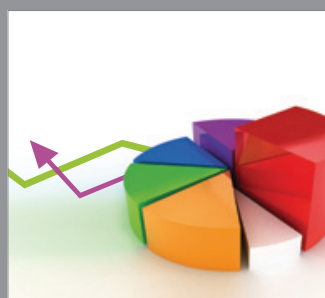

ournal of

Probability and Statistics

Promensencen
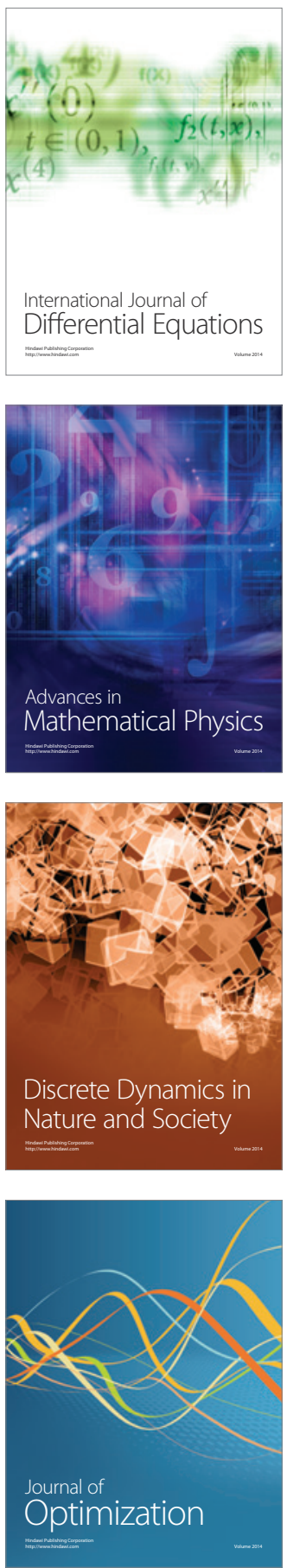